\title{
The evidence for improving housing to reduce malaria: a systematic review and meta-analysis
}

Lucy S Tusting $^{1 *+}$, Matthew M Ippolito ${ }^{2 \dagger}$, Barbara A Willey ${ }^{3}$, Immo Kleinschmidt ${ }^{3}$, Grant Dorsey ${ }^{4}$, Roly D Gosling ${ }^{5}$ and Steve W Lindsay ${ }^{6}$

\begin{abstract}
Background: The global malaria burden has fallen since 2000, sometimes before large-scale vector control programmes were initiated. While long-lasting insecticide-treated nets and indoor residual spraying are highly effective interventions, this study tests the hypothesis that improved housing can reduce malaria by decreasing house entry by malaria mosquitoes.
\end{abstract}

Methods: A systematic review and meta-analysis was conducted to assess whether modern housing is associated with a lower risk of malaria than traditional housing, across all age groups and malaria-endemic settings. Six electronic databases were searched to identify intervention and observational studies published from 1 January, 1900 to 13 December, 2013, measuring the association between house design and malaria. The primary outcome measures were parasite prevalence and incidence of clinical malaria. Crude and adjusted effects were combined in fixed- and random-effects meta-analyses, with sub-group analyses for: overall house type (traditional versus modern housing); screening; main wall, roof and floor materials; eave type; ceilings and elevation.

Results: Of 15,526 studies screened, 90 were included in a qualitative synthesis and 53 reported epidemiological outcomes, included in a meta-analysis. Of these, 39 (74 \%) showed trends towards a lower risk of epidemiological outcomes associated with improved house features. Of studies assessing the relationship between modern housing and malaria infection $(n=11)$ and clinical malaria $(n=5)$, all were observational, with very low to low quality evidence. Residents of modern houses had $47 \%$ lower odds of malaria infection compared to traditional houses (adjusted odds ratio (OR) $0^{\circ} 53,95 \%$ confidence intervals (Cl) $0^{\circ} 42-0^{\circ} 67, p<0^{\circ} 001$, five studies) and a $45-65 \%$ lower odds of clinical malaria (case-control studies: adjusted OR $0^{\circ} 35,95 \% \mathrm{Cl} 0^{\circ} 20-0^{\circ} 62, p<0^{\circ} 001$, one study; cohort studies: adjusted rate ratio $0^{\circ} 55,95 \% \mathrm{Cl} 0^{\circ} 36-0^{\circ} 84, p=0^{\circ} 005$, three studies). Evidence of a high risk of bias was found within studies.

Conclusions: Despite low quality evidence, the direction and consistency of effects indicate that housing is an important risk factor for malaria. Future research should evaluate the protective effect of specific house features and incremental housing improvements associated with socio-economic development.

Keywords: Malaria, Plasmodium falciparum, Vector, Anopheles gambiae, House, Eaves, Socio-economic

\section{Background}

Despite considerable advances in malaria control since 2000 , with a $30 \%$ fall in incidence in all age groups worldwide, the disease remains a major global public health problem with an estimated 198 million cases in 2013 [1]. Reductions have been achieved mainly through extensive long-lasting insecticide-treated net

\footnotetext{
* Correspondence: lucy.tusting@lshtm.ac.uk

${ }^{\dagger}$ Equal contributors

'Department of Disease Control, London School of Hygiene \& Tropical Medicine, London WC1E 7HT, UK

Full list of author information is available at the end of the article
}

(LLIN) distribution and indoor residual spraying (IRS) campaigns. However, the future success of these interventions may be undermined by the spread of insecticide-resistant mosquitoes [2], creating a need for supplementary interventions not reliant on current insecticides. Interestingly, in some locations malaria has declined before intervention scale-up, suggesting additional causes of the reduction [3,4]. Since malaria is a disease of poverty and the environment, there is increasing interest in the potential contribution of socioeconomic development to malaria control [5], and in

\section{Biomed Central}

(c) 2015 Tusting et al.; licensee BioMed Central. This is an Open Access article distributed under the terms of the Creative Commons Attribution License (http://creativecommons.org/licenses/by/4.0), which permits unrestricted use, distribution, and reproduction in any medium, provided the original work is properly credited. The Creative Commons Public Domain Dedication waiver (http://creativecommons.org/publicdomain/zero/1.0/) applies to the data made available in this article, unless otherwise stated. 
coordinating with sectors outside health, including agriculture, water and sanitation, education, city planning and housing, to meet long-term sustainable development goals [6].

Housing improvements, traditionally a key pillar of public health, remain underexploited in malaria control. Yet in sub-Saharan Africa (SSA), where up to 80-100 \% of malaria transmission occurs indoors at night, the home can be a place of high risk [7]. House screening was the first intervention trialed in Italy after the link between malaria and mosquitoes was discovered [8]. Screening homes was subsequently shown to reduce malaria risk in India, South Africa and the USA [6] and better housing contributed to malaria elimination in the USA and Europe [9]. More recent studies indicate that well-built, modern housing can be protective in many tropical countries [10] and that simple features, including closed eaves (the gap between the top of the wall and the over-hanging roof), brick walls, tiled or metal roofs, or ceilings can reduce mosquito house entry [6]. In a randomized-controlled trial (RCT) in The Gambia, untreated door and window screens and closed eaves halved the prevalence of anaemia in children [11].

Ninety per cent of malaria deaths in five year-olds occur in Africa, the economy of which is rapidly growing, with a $6 \%$ annual increase in gross domestic product expected until 2025 [12]. Increased personal wealth is precipitating continent-wide housing improvements, such as the replacement of traditional thatch with metal and tiled roofs (Fig. 1). The expanding population, expected to triple to 1.23 billion by 2050 , also needs accommodating, with an estimated 144 million new houses required by 2030 in rural areas alone [13]. This economic and cultural transition presents an opportunity

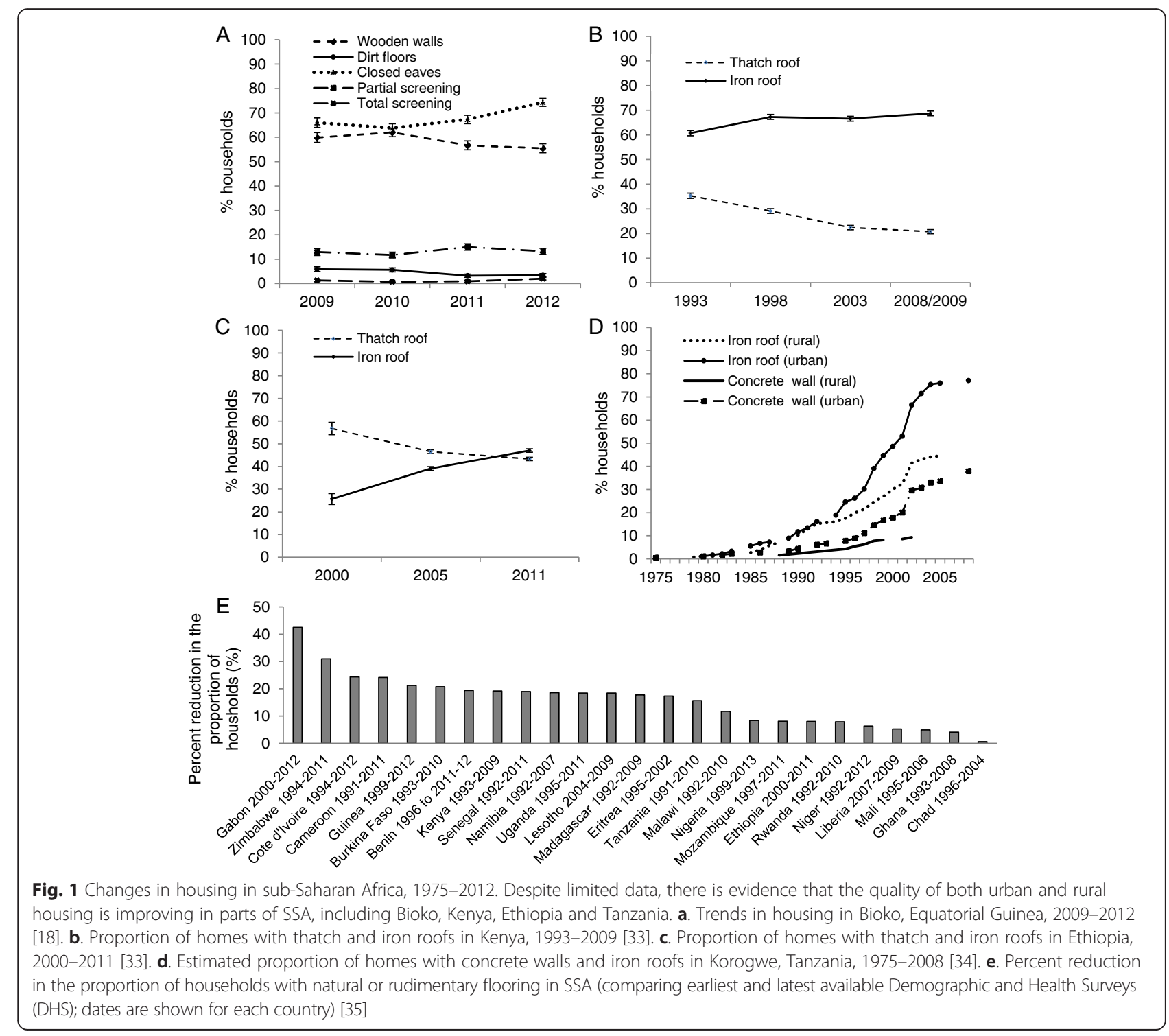


to document and influence incremental housing improvements that might protect against malaria, and to build healthy homes.

Yet despite the historical precedent for improving housing to control malaria, few rigorously conducted studies exist. Furthermore, the evidence on housing and malaria has not been systematically characterized, with no specific evaluation of the size and consistency of the direction of effect, nor the quality of the evidence. The recent Multisectoral Action Framework for Malaria [14] emphasizes throughout the need for good housing, yet there is a paucity of evidence supporting this recommendation and uncertainty about how to select, scale-up and sustain intervention [6]. Here the potential for modern house construction to reduce malaria risk was evaluated. Specifically, the first systematic review and meta-analysis was conducted to assess whether 'modern' homes are associated with reduced exposure to infectious bites, malaria infection and clinical malaria in people of all ages in malaria-endemic regions, compared to 'traditional' homes. Since few intervention studies exist, observational study designs were also included. The study aimed first to characterize all published and unpublished data and second to assess the strength and quality of these data, in order to rigorously evaluate the evidence for the impact of housing improvements on malaria.

\section{Methods}

Recommendations of the Preferred Reporting Items for Systematic Reviews and Meta-analysis of Observational Studies in Epidemiology groups were followed $[15,16]$. The study is registered with the International Prospective Register of Systematic Reviews [17]. The study aimed to compare modern with traditional homes in any malaria-endemic settings. In SSA, traditional homes were considered to have mud walls, thatched roofs and earth floors, except in areas of exceptionally high rainfall including Equatorial Guinea, where concrete or wood is the basic wall material [18]. Traditional homes were considered to have mud or stone walls, thatched, wood or mud roofs, and earth floors in North Africa [19], wood or bamboo walls, thatched roofs and earth or wooden floors in Southeast and South Asia [20], and adobe or mud and wood walls, thatched roofs and earth floors in South America [21]. Universally, traditional homes were considered to have open eaves, no ceiling and no screening.

\section{Eligibility criteria}

Studies were included with participants of any ages (excluding migrants, displaced people or military) and conducted in real (not experimental) houses, that compared modern with traditional house features and that measured any outcomes of interest. Both observational and intervention study designs were included: (1) case-control; (2) cohort; (3) cross-sectional studies; (4) randomizedcontrolled trials (RCTs); (5) controlled before-and-after studies, if arms were comparable at baseline and there was at least one unit per arm; (6) cross-over studies, if there were at least one unit per arm; and, (7) interrupted time-series studies. Studies were excluded if arm follow-up periods differed.

Epidemiological outcomes in human subjects were: clinical malaria (fever with parasitaemia confirmed by microscopy or rapid diagnostic test (RDT), in any age group); malaria infection (confirmed by microscopy or RDT, in any age group); and, anaemia in children aged under 11 years. Entomological outcomes were: entomological inoculation rate (EIR, the estimated number of bites by infectious mosquitoes per person per time period, measured directly using human baits or indirectly using light traps or other methods); human biting rate (HBR, the number of mosquitoes per person per time period); and, indoor density of adult vector mosquitoes (number of mosquitoes per house or person).

\section{Search strategy and data extraction}

PubMed, Embase, LILACS, the Meta-Register of Controlled Trials, Cochrane Infectious Diseases Group Specialized Register, and Cochrane Central Register of Controlled Trials were searched with no language restrictions, using specified search terms (Additional file 1) to identify studies published from 1 January, 1900 to 13 December, 2013. The following databases were searched: US Armed Forces Pest Management Board online database (1900-1947) and proceedings of the MIM PanAfrican Malaria Conferences (2005 and 2013), American Society of Tropical Medicine and Hygiene (2004-2013) and Society for Vector Ecology (2010-2012). Reference lists of identified studies were searched. Authors were contacted for additional references. LST and MI independently screened titles and abstracts before screening the full text of relevant studies using a standard form. Disagreements were resolved by SWL.

\section{Data extraction}

Study characteristics (participants, sampling, exposures, comparisons, outcomes, study design, setting, sample size, follow-up period, vector(s), LLIN and IRS coverage, transmission intensity, and funding) were extracted by LST and a $10 \%$ sub-sample randomly selected for validation (MI). Study authors were contacted for missing data.

\section{Risk of bias of and quality of evidence}

Risk of bias for RCTs, controlled before-and-after studies, cross-over studies and interrupted time-series studies was assessed using the Effective Practice and Organization of 
Care (EPOC) tool [22], and for case-control, cohort and cross-sectional studies using the Newcastle-Ottawa Scale [23]. Risk of bias across studies (publication bias) was assessed using funnel plots and Egger's test for funnel plot asymmetry [24]. Quality and strength of the evidence were evaluated for the main comparison (modern versus traditional homes) using the Grading of Recommendations, Assessment, Development and Evaluation (GRADE) approach [25].

\section{Data analysis}

Analyses were structured first by house feature, second by outcome and third by study design. All eligible studies were included in a qualitative synthesis. Studies were also included in a quantitative analysis, comparing modern with traditional house features, if crude or adjusted odds ratios (ORs) or rate ratios (RRs) with $95 \%$ confidence intervals $(\mathrm{CI})$, or sufficient data to calculate crude effects, were reported. Specifically, epidemiological data were combined in meta-analysis and entomological data presented in tables. Analyses were done in Stata13 and RevMan5.

\section{Epidemiological data}

Study effects were combined in the meta-analysis using the generic inverse variance method, which assigns each effect a weight equal to the inverse of its variance. Pooled ORs or RRs were calculated using fixed-effects meta-analysis where significant heterogeneity was not detected and random effects where significant heterogeneity was found $\left(\mathrm{I}^{2}>50 \%\right)$. Separate meta-analyses were done for crude and adjusted results.

\section{Entomological data}

Data and study characteristics were presented in tables. Where no effect measure was reported the crude effect was calculated as the ratio of the outcomes in the treatment and control groups. Ninety-five per cent CIs were calculated by estimating the standard errors of the outcomes from their stated $95 \%$ CI. Where $95 \%$ CIs of outcomes were non-symmetrical, it was assumed that standard errors and CIs were calculated on log-transformed values.

\section{Results}

\section{Search results}

The search yielded 15,526 studies after removing duplicates (Fig. 2). Ninety met the inclusion criteria, of which 18 were included in the qualitative synthesis only and 72 were included in the quantitative analysis (Additional file 2). Of these 72 studies, 53 reported epidemiological outcomes (included in the meta-analysis) and 25 reported entomological outcomes (presented in Tables).

\section{Study characteristics}

The six intervention studies dated from 2009 to 2013. All were conducted in rural SSA, using house screening as the intervention. One study, a cluster RCT (cRCT) in The Gambia, collected both epidemiological and entomological outcomes [11] and was included in the metaanalysis. Five studies collected entomological data only: three pilot RCTs in Ethiopia, Mozambique and Tanzania [26-28], one randomized cross-over trial in The Gambia [29], and one non-randomized, cross-over trial in Tanzania [30]. The 84 observational studies, dating from 1935 to 2015, had cross-sectional $(n=39)$, cohort $(n=30)$, and case-control $(n=15)$ designs. These were conducted mainly in SSA $(n=58)$ and Asia $(n=13)$ and largely in rural settings $(n=62)$ (Additional file 2$)$. In the 53 observational studies included in the meta-analysis, comparisons included modern versus traditional housing $(n=15)$; modern versus traditional wall $(n=22)$, roof $(n=18)$, and floor $(n=4)$ materials and closed versus open eaves $(n=11)$.

\section{Risk of bias and quality of the evidence}

High risk of bias was found across numerous domains of the EPOC risk of bias for intervention studies, particularly for allocation concealment, length of follow-up and blinding (Additional file 3). Risk of bias within individual case-control, cross-sectional and cohort studies was generally high (Additional file 3). Across studies, there was no evidence of publication bias in the meta-analysis of house type and malaria infection (Additional file 4), with no evidence of funnel plot asymmetry (bias coefficient $0.52,95 \% \mathrm{CI}-1.61$ to $2.65, p=0.60$ ). There were insufficient studies to test for asymmetry in the metaanalysis of house type and clinical malaria. GRADE quality of the evidence for the main comparison, modern versus traditional housing, ranged from very low to low (Table 1).

\section{Modern versus traditional housing}

Residents of modern homes had lower odds of malaria infection than residents of traditional homes (crude OR $0^{\circ} 46,95 \%$ CI $0^{\circ} 33-0^{\circ} 62, p<0^{\circ} 001$, nine studies, low quality evidence; adjusted OR $0^{\circ} 53,95 \%$ CI $0^{\circ} 42-0^{\circ} 67$, $p<0^{\circ} 001$, five studies, very low quality evidence) (Fig. 3, Table 2). Modern homes were associated with lower odds and incidence rate of clinical malaria (case-control and cross-sectional studies: crude OR $0^{\circ} 32,95 \%$ CI $0^{\circ} 19-0^{\circ} 54, p<0^{\circ} 001$, one study, very low quality evidence; adjusted OR $0^{\circ} 35,95 \%$ CI $0^{\circ} 20-0^{\circ} 62, p<0^{\circ} 001$, one study, very low quality evidence; cohort studies: crude RR $0^{\circ} 22,95 \%$ CI $0^{\circ} 14-0^{\circ} 35, p<0^{\circ} 001$, three studies, low quality evidence; adjusted RR $0^{\circ} 55,95 \% \mathrm{CI}$ $0^{\circ} 36-0^{\circ} 84, p=0.005$, three studies, very low quality evidence) (Fig. 4). In seven studies with entomological 


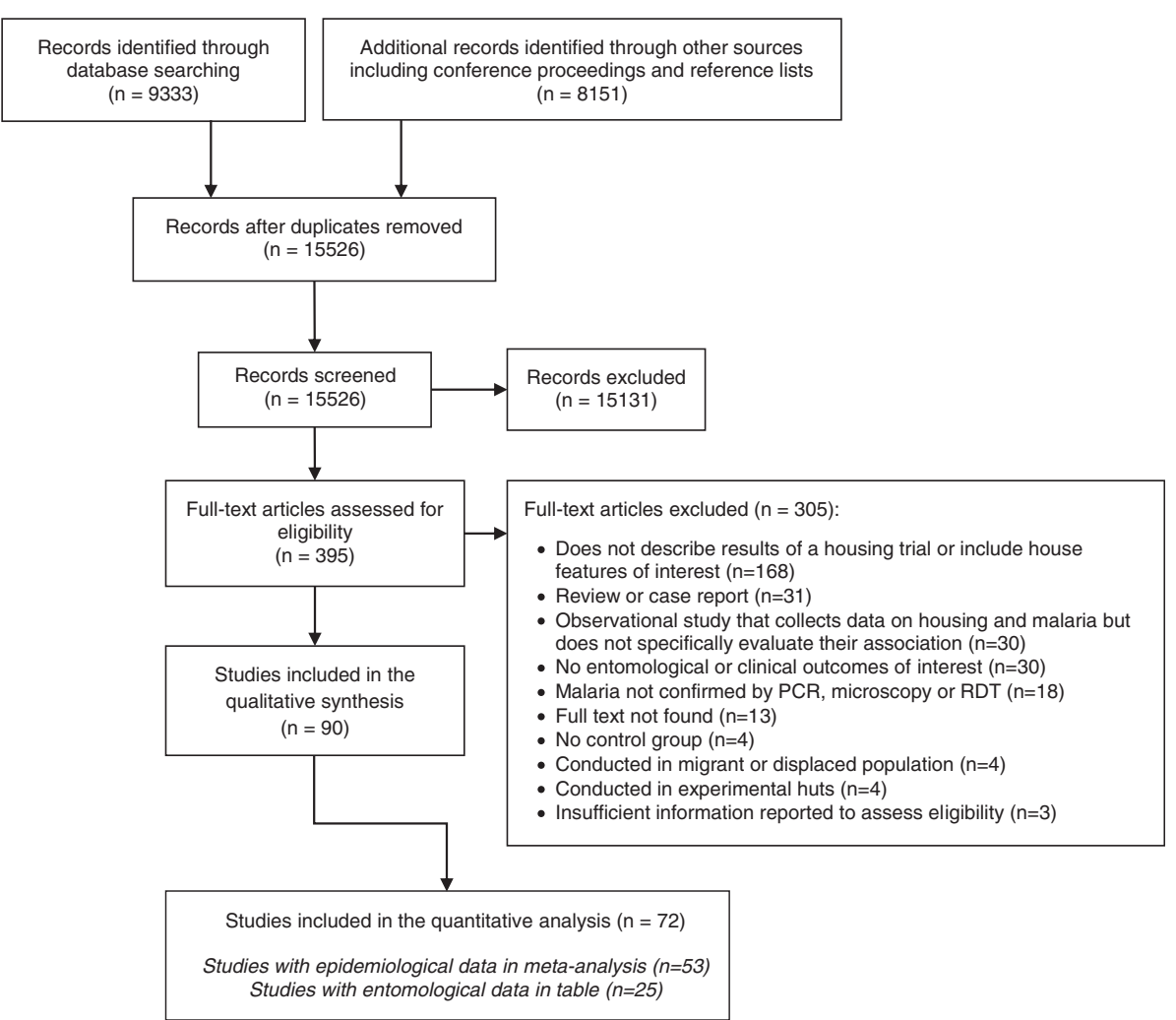

Fig. 2 Study selection

outcomes, modern housing was associated with no effect to a $66 \%$ reduction in density of adult anophelines (Additional file 5).

\section{House screening}

In one cRCT in The Gambia, full or ceiling screening reduced anaemia in children by $48 \%$ (adjusted OR $0^{\circ} 52$, $95 \%$ CI $0^{\circ} 34-0^{\circ} 80, p=0^{\circ} 003$ ), with no effect on malaria infection. Screening was not consistently associated with lower odds of anaemia in children or malaria infection in seven case-control, cross-sectional and cohort studies, but was associated with a lower incidence of clinical malaria in three cohort studies (Table 2).

\section{Modern versus traditional wall, roof and floor materials}

Modern wall materials were associated with an approximately quarter reduction in the odds of malaria infection, although results were inconsistent for incidence of clinical malaria. Modern roof materials were not consistently associated with reduced odds of infection, but were associated with up to a two thirds reduction in the incidence of clinical malaria. There was inconsistent evidence that modern floor materials gave protection against any epidemiological outcome (Table 2).

\section{Eaves, ceilings and house elevation}

Closed eaves were associated with a quarter reduction in the odds of malaria infection and a quarter to a half reduction in clinical malaria in five case-control and cross-sectional studies and two cohort studies. The presence (versus absence) of a ceiling was associated with a third reduction in the odds of clinical malaria. In one cross-sectional study, house elevation was not associated with the odds of malaria infection (Table 2).

\section{Discussion}

In this systematic review and meta-analysis to assess whether modern housing is associated with a lower risk of malaria than traditional housing, 84 observational and six intervention studies were included. In eleven case-control, cohort and cross-sectional studies in East Timor, Egypt, Ethopia, Greece, Malawi, Sri Lanka, Thailand, Uganda, and Yemen, the odds of malaria infection were halved in modern versus traditional homes. In one case-control study in Mexico, the odds of clinical malaria were reduced by two thirds. In four cohort studies in Ethiopia, Tanzania and Uganda, the incidence of clinical malaria was halved in modern versus traditional homes.

Although house screening was the first intervention trialled against malaria [8], few intervention studies have 
Table 1 GRADE quality of evidence for the association between modern housing and clinical malaria outcomes

\begin{tabular}{|c|c|c|c|c|c|c|c|c|}
\hline \multirow[t]{2}{*}{ Outcomes } & \multicolumn{2}{|c|}{ Summary of findings } & \multicolumn{5}{|c|}{ Quality of the evidence } & \multirow[t]{2}{*}{ Overall quality of the evidence (GRADE) } \\
\hline & $\begin{array}{l}\text { Relative effect } \\
(95 \% \mathrm{Cl})\end{array}$ & $\begin{array}{l}\text { No. participants } \\
\text { (studies) }\end{array}$ & Risk of bias & Inconsistency & Indirectness & Imprecision & Publication bias & \\
\hline $\begin{array}{l}\text { Malaria infection: Case-control, cross-sectional } \\
\text { and cohort studies (crude OR) }\end{array}$ & $\begin{array}{l}\text { OR } 0^{\circ} 46 \\
\left(0^{\circ} 33-0^{\circ} 62\right)\end{array}$ & 22,700 (9 studies) & Serious $^{1}$ & $\begin{array}{l}\text { No serious } \\
\text { inconsistency }\end{array}$ & $\begin{array}{l}\text { No serious } \\
\text { indirectness }^{3}\end{array}$ & $\begin{array}{l}\text { No serious } \\
\text { imprecision }^{4}\end{array}$ & Undetected $^{5}$ & $\begin{array}{l}\text { LOW } 1,2,3,4,5,6,7 \text { due to risk of bias, large } \\
\text { effect }\end{array}$ \\
\hline $\begin{array}{l}\text { Malaria infection: Case-control, cross-sectional } \\
\text { and cohort studies (adjusted OR) }\end{array}$ & $\begin{array}{l}\text { OR } 0^{\circ} 53 \\
\left(0^{\circ} 42-0^{\circ} 67\right)\end{array}$ & 3949 (5 studies) & Serious $^{1}$ & $\begin{array}{l}\text { No serious } \\
\text { inconsistency }\end{array}$ & $\begin{array}{l}\text { No serious } \\
\text { indirectness }^{9}\end{array}$ & $\begin{array}{l}\text { No serious } \\
\text { imprecision }^{4}\end{array}$ & Undetected $^{5}$ & VERY LOW $1,4,5,7,8,9,10$ due to risk of bias \\
\hline $\begin{array}{l}\text { Clinical malaria: Case-control and } \\
\text { cross-sectional studies (crude OR) }\end{array}$ & $\begin{array}{l}\text { OR } 0^{\circ} 32 \\
\left(0^{\circ} 19-0^{\circ} 54\right)\end{array}$ & 357 (1 study) & Serious $^{1}$ & $\begin{array}{l}\text { No serious } \\
\text { inconsistency }\end{array}$ & Serious $^{12}$ & $\begin{array}{l}\text { No serious } \\
\text { imprecision }^{4}\end{array}$ & Undetected $^{13}$ & $\begin{array}{l}\text { VERY LOW } \\
\text { indirectness, large effect }\end{array}$ \\
\hline $\begin{array}{l}\text { Clinical malaria: Case-control and } \\
\text { cross-sectional studies (adjusted OR) }\end{array}$ & $\begin{array}{l}\text { OR } 0^{\circ} 35 \\
\left(0^{\circ} 20-0^{\circ} 62\right)\end{array}$ & 357 (1 study) & Serious $^{1}$ & $\begin{array}{l}\text { No serious } \\
\text { inconsistency }\end{array}$ & Serious $^{12}$ & $\begin{array}{l}\text { No serious } \\
\text { imprecision }^{4}\end{array}$ & Undetected $^{13}$ & $\begin{array}{l}\text { VERY LOW } 14,6,6,11,12,13 \text { due to risk of bias, } \\
\text { indirectness, large effect }\end{array}$ \\
\hline Clinical malaria: Cohort studies (crude RR) & $\begin{array}{l}\text { RR } 0^{\circ} 22 \\
\left(0^{\circ} 14-0^{\circ} 35\right)\end{array}$ & 1653 (3 studies) & Serious $^{1}$ & $\begin{array}{l}\text { No serious } \\
\text { inconsistency }{ }^{14}\end{array}$ & Serious $^{15}$ & $\begin{array}{l}\text { No serious } \\
\text { imprecision }^{4}\end{array}$ & Undetected ${ }^{13}$ & $\begin{array}{l}\text { LOW } 1,4,7,13,14,15,16 \text { due to risk of bias, } \\
\text { indirectness, large effect }\end{array}$ \\
\hline Clinical malaria: Cohort studies (adjusted RR) & $\begin{array}{l}\text { RR } 0^{\circ} 55 \\
\left(0^{\circ} 36-0^{\circ} 84\right)\end{array}$ & 2237 (3 studies) & Serious $^{1}$ & $\begin{array}{l}\text { No serious } \\
\text { inconsistency }{ }^{17}\end{array}$ & Serious ${ }^{15}$ & $\begin{array}{l}\text { No serious } \\
\text { imprecision }^{4}\end{array}$ & Undetected ${ }^{13}$ & $\begin{array}{l}\text { VERY LOW } 1,4,13,15,17,18 \text { due to risk of bias, } \\
\text { indirectness }\end{array}$ \\
\hline
\end{tabular}

Patient or population: People of all ages living in malaria-endemic regions

Settings: East Timor, Egypt, Ethiopia, Greece, Malawi, Mexico, Sri Lanka, Tanzania, Thailand, Uganda and Yemen

Intervention: modern (versus traditional) housing

GRADE Working Group grades of evidence: High quality: Further research is very unlikely to change confidence in the estimate of effect. Moderate quality: Further research is likely to have an important impact on

confidence in the estimate of effect and may change the estimate. Low quality: Further research is very likely to have an important impact on confidence in the estimate of effect and is likely to change the estimate.

Very low quality: The estimate is very uncertain

${ }^{1}$ Downgraded by 1 for serious risk of bias: All studies were non-randomized and observational

${ }^{2}$ No serious inconsistency: All nine studies observed a protective effect of modern housing, compared to traditional housing. The smallest effect was a $28 \%$ reduction in the odds of malaria infection

${ }^{3}$ No serious indirectness: These nine studies were conducted in a variety of sites, both urban and rural, in settings across sub-Saharan Africa, Asia and Europe. The findings are generalizable elsewhere

${ }^{4}$ No serious indrectness: These nine studies were conducted in a variety of sites, both urban and

${ }^{5}$ Publication bias not detected: Egger's test for bias in crude results found no evidence funnel plot asymmetry (bias coefficient $0.52,95 \% \mathrm{Cl}-1.61-2.65, \mathrm{p}=0.60$ )

'Publication bias not detected: Egger's test for bias in crude results
6 Upgraded by 1 for large effect: OR lies within the range 0 to 0.5

${ }^{6}$ Upgraded by 1 for large effect: OR lies within the range 0 to 0.5
${ }^{7}$ No evidence that residual confounding would reduce the demonstrated effect: no significant difference between crude and adjusted effects

No evidence that residual confounding would reduce the demonstrated effect: no significant difference between crude and adjusted effects
${ }^{8}$ No serious inconsistency: All five studies observed a protective effect of modern housing, compared to traditional housing. The smallest effect was a $27 \%$ reduction in the odds of malaria infection

${ }^{9} \mathrm{No}$ serious indirectness: These five studies were conducted in a variety of sites, both urban and rural, in sub-Saharan Africa and Asia. The findings are generalizable elsewhere

${ }^{10} \mathrm{No}$ large effect: Odds Ratio does not fall into the range 0 to 0.5

${ }^{11}$ No serious inconsistency: only one study

${ }^{12}$ Downgraded by 1 for indirectness: only one study was included, which was conducted in rural Mexico and the findings may not be generalizable elsewhere

${ }^{13}$ Publication bias not detected: insufficient studies to construct funnel plots

${ }^{14} \mathrm{No}$ serious inconsistency: all three studies observed a protective effect of modern housing, compared to traditional housing. The smallest effect was a $53 \%$ reduction in incidence of clinical malaria

${ }_{115}^{15}$ owngraded by 1 for serious indirectness: all studies were conducted in rural sub-Saharan Africa. The results may not be generalizable to other settings

${ }^{16}$ Upgraded by 2 for very large effect: Rate ratio and $95 \%$ confidence intervals lie within the range 0 to $0{ }^{\circ} 5$

${ }^{17}$ No serious inconsistency: all three studies observed a protective effect of modern housing, compared to traditional housing. The smallest effect was a $25 \%$ reduction in the incidence of clinical malaria

${ }^{18} \mathrm{No}$ large effect: RR does not fall into the range 0 to $0^{\circ} 5$ 


\begin{tabular}{|c|c|c|c|c|c|c|}
\hline Study or Subgroup & log[Odds Ratio] & LE & Weight & $\begin{array}{l}\text { Odds Ratio } \\
\text { IV, Random, } 95 \% \mathrm{Cl}\end{array}$ & $\begin{array}{r}\text { Odds } \\
\text { IV, Rando }\end{array}$ & $\begin{array}{l}\text { s Ratio } \\
\text { om, } 95 \% \mathrm{Cl}\end{array}$ \\
\hline \multicolumn{7}{|c|}{ 1.1.1 Case-control, cross-sectional and cohort studies (crude odds ratio) } \\
\hline Al-Makhlafi 2011 YEM (1) & -0.328504 & 0.382809 & $9.5 \%$ & $0.72[0.34,1.52]$ & & \\
\hline Barber 1935 GRC (2) & -0.554898 & 0.320402 & $11.4 \%$ & $0.57[0.31,1.08]$ & & \\
\hline Butraporn 1986 THA (3) & -1.347074 & 0.133859 & $18.4 \%$ & $0.26[0.20,0.34]$ & $\leftarrow$ & \\
\hline Dahesh 2009 EGY (4) & -0.392042 & 0.459362 & $7.7 \%$ & $0.68[0.27,1.66]$ & & \\
\hline de Alemida 2010 TLS (5) & -1.407117 & 1.458633 & $1.1 \%$ & $0.24[0.01,4.27]$ & & \\
\hline Osterbauer 2012 UGA (6) & -1.514128 & 0.525624 & $6.4 \%$ & $0.22[0.08,0.62]$ & & \\
\hline van der Hoek 2003 LKA (7) & -0.875469 & 0.195404 & $16.0 \%$ & $0.42[0.28,0.61]$ & & \\
\hline Wolff $2001 \mathrm{MWI}(8)$ & -0.371064 & 0.331932 & $11.0 \%$ & $0.69[0.36,1.32]$ & & \\
\hline $\begin{array}{l}\text { Woyessa } 2013 \mathrm{ETH}(9) \\
\text { Subtotal }(95 \% \mathrm{Cl})\end{array}$ & -0.634878 & 0.13098 & $\begin{array}{r}18.5 \% \\
100.0 \%\end{array}$ & $\begin{array}{l}0.53[0.41,0.69] \\
0.46[0.33,0.62]\end{array}$ & & \\
\hline \multicolumn{7}{|c|}{$\begin{array}{l}\text { Heterogeneity: } \mathrm{Tau}^{2}=0.12 ; \mathrm{Chi}^{2}=23.85, \mathrm{df}=8(P=0.002) ; \mathrm{I}^{2}=66 \% \\
\text { Test for overall effect: } Z=4.92(P<0.00001)\end{array}$} \\
\hline \multicolumn{7}{|c|}{ 1.1.2 Case-control, cross-sectional and cohort studies (adjusted odds ratio) } \\
\hline de Beaudrap 2001 UGA (10) & -0.494296 & 0.202705 & $28.7 \%$ & $0.61[0.41,0.91]$ & & \\
\hline Osterbauer 2012 UGA (11) & -1.386294 & 0.521251 & $4.9 \%$ & $0.25[0.09,0.69]$ & & \\
\hline van der Hoek 2003 LKA (12) & -0.553885 & 0.21463 & $25.9 \%$ & $0.57[0.38,0.88]$ & & \\
\hline Wanzirah 2015 UGA (13) & -0.820981 & 0.195404 & $30.5 \%$ & $0.44[0.30,0.65]$ & & \\
\hline $\begin{array}{l}\text { Wolff } 2001 \mathrm{MWI}(14) \\
\text { Subtotal }(95 \% \mathrm{Cl})\end{array}$ & -0.314711 & 0.360684 & $\begin{array}{r}10.0 \% \\
100.0 \%\end{array}$ & $\begin{array}{l}0.73[0.36,1.48] \\
0.53[0.42,0.67]\end{array}$ & & \\
\hline \multicolumn{7}{|c|}{$\begin{array}{l}\text { Heterogeneity: } \mathrm{Tau}^{2}=0.01 ; \mathrm{Chi}^{2}=4.40, \mathrm{df}=4(P=0.35) ; \mathrm{I}^{2}=9 \% \\
\text { Test for overall effect: } Z=5.44(P<0.00001)\end{array}$} \\
\hline \multicolumn{7}{|c|}{ Test for subgroup differences: $\mathrm{Chi}^{2}=0.56, \mathrm{df}=1(P=0.45), \mathrm{I}^{2}=0 \%$} \\
\hline \multicolumn{7}{|c|}{$\begin{array}{l}\text { Fig. } 3 \text { Meta-analysis of the association between modern housing and malaria infection. Pooled effects from random-effects meta-analyses for } \\
\text { crude }\left(1^{\circ} 1^{\circ} 1\right) \text { and adjusted }\left(1^{\circ} 1^{\circ} \text { 2) results are shown. Studies are divided into sub-groups by study design. Error bars show } 95 \% \text { Cls; df = degrees }\right. \\
\text { of freedom. 1. Al-Makhlafi } 2011 \text { YEM: Good vs poor house quality; } 2 \text {. Barber } 1935 \text { GRC: Modern (tiled roof, ceiling) vs traditional (thatched roof, reed } \\
\text { or no ceiling); 3. Butraporn } 1935 \text { THA: Permanent vs semi-permanent or temporary; 4. Dahesh } 2009 \text { EGY: Painted brick walls and cement ceilings vs } \\
\text { mud walls and wood or mud ceilings; } 5 \text {. de Alemida } 2010 \text { TLS: Complete vs incomplete house; } 6 \text {. Osterbauer } 2012 \text { UGA: Modern (iron roof, burnt brick } \\
\text { or cement walls and cement floor) vs traditional; } 7 \text {. van der Hoek } 2003 \text { LKA: Modern (brick walls and permanent roof material) vs traditional (mud walls } \\
\text { or thatched roof); 8. Wolff } 2001 \text { MWl: Modern vs traditional; } 9 \text {. Woyessa } 2013 \text { ETH: Good vs dilapidated house, 10. de Beaudrap } 2001 \text { UGA: Brick walls } \\
\text { and iron roof vs mud walls and thatched roof (OR adjusted for age, weight, socio-economic status, education, altitude, ITNs), } 11 \text {. Osterbauer } 2012 \text { UGA: } \\
\text { Modern (iron roof, burnt brick or cement walls and cement floor) vs traditional (OR adjusted for age, HIV-exposure, enrolment period, gender, mother's } \\
\text { age, prophylaxis); } 12 \text {. van der Hoek } 2003 \text { LKA: Modern (brick walls and permanent roof material) vs traditional (mud walls or thatched roof) (OR adjusted } \\
\text { for age, gender, distance to stream, distance to cattle shed, coil use, ITNs, IRS); 13. Wanzirah } 2015 \text { UGA: Modern (cement, wood or metal wall; tiled or } \\
\text { metal roof and closed eaves) vs traditional (OR adjusted for age, gender, study site, household wealth); } 14 \text {. Wolff } 2001 \text { MWl: Modern vs traditional (OR } \\
\text { adjusted for water source, occupation, education, malaria knowledge, waste disposal method) }\end{array}$} \\
\hline
\end{tabular}

rigorously evaluated the effect of housing on malaria. Observational studies were therefore also included here, which were most likely subject to selection and measurement bias, low comparability between groups, residual confounding by wealth [5], and geographical clustering of socio-economic status, house design and malaria. Although we found no evidence of publication bias across studies, we had limited power to detect publication bias due to the relatively small number of studies included [24]. Therefore it is highly possible that publication bias, selective outcome reporting, small-study effects, or selective analysis reporting were present across studies. Overall GRADE quality of evidence was judged to be 'very low' to 'low', indicating considerable uncertainty in the estimated effects. Despite this, the relative consistency of the size and direction of effect across studies and settings indicates some protection by modern housing, compared to traditional homes, in urban and rural settings in Africa, Asia and South America. Specifically, wall and roof materials other than traditional wood, mud and thatch, and modern house designs encompassing closed eaves, screened doors and windows, and ceilings, may help reduce mosquito house entry and malaria transmission, and therefore merit further field evaluation.

Good housing can help protect by blocking the entry routes of malaria vectors, which vary by species and region. Overall, the reduced prevalence and incidence of malaria in modern versus traditional homes indicates that this classification was a good proxy for overall ease of entry by mosquitoes across different settings. Closed eaves are likely to be protective in sub-Saharan Africa since the primary African vector Anopheles gambiae s.l. locates hosts by following odour plumes close to the ground and flying upwards when a vertical surface is reached. Open eaves then funnel mosquitoes inside [29]. 
Table 2 Summary of findings of meta-analyses of the association between specific house features and malaria

\begin{tabular}{|c|c|c|c|c|}
\hline Comparison & Outcome & Study design & Total studies & $\begin{array}{l}\text { Effect estimate } \\
(95 \% \mathrm{Cl})\end{array}$ \\
\hline \multirow[t]{6}{*}{1 Modern versus traditional housing } & 1.1 Malaria infection & 1.1.1 Case-control, cross-sectional and cohort studies (crude OR) & 9 & $0.46[0.33,0.62]$ \\
\hline & & 1.1.2 Case-control, cross-sectional and cohort studies (adjusted OR) & 5 & $0.53[0.42,0.67]$ \\
\hline & 1.2 Clinical malaria & 1.2.1 Case-control and cross-sectional studies (crude OR) & 1 & $0.32[0.19,0.54]$ \\
\hline & & 1.2.2 Case-control and cross-sectional studies (adjusted OR) & 1 & $0.35[0.20,0.62]$ \\
\hline & & 1.2.3 Cohort studies (crude RR) & 3 & $0.22[0.14,0.35]$ \\
\hline & & 1.2.4 Cohort studies (adjusted RR) & 3 & $0.55[0.36,0.84]$ \\
\hline \multirow[t]{9}{*}{2 Screening ${ }^{1}$} & 2.1 Anaemia in children aged 0-11 years & 2.1.1 Randomized controlled trials (adjusted OR) & 1 & $0.52[0.34,0.80]$ \\
\hline & & 2.1.2 Case-control, cross-sectional and cohort studies (crude OR) & 2 & $0.65[0.33,1.30]$ \\
\hline & & 2.1.3 Case-control, cross-sectional and cohort studies (adjusted OR) & 1 & $0.56[0.24,1.27]$ \\
\hline & 2.2 Malaria infection & 2.2.1 Randomized controlled trials (adjusted OR) & 1 & $0.95[0.63,1.43]$ \\
\hline & & 2.2.2 Case-control, cross-sectional and cohort studies (crude OR) & 5 & $0.35[0.13,0.98]$ \\
\hline & & 2.2.3 Case-control, cross-sectional and cohort studies (adjusted OR) & 2 & $0.93[0.82,1.05]$ \\
\hline & 2.3 Clinical malaria & 2.3.1 Case-control and cross-sectional studies (crude OR) & 1 & $1.16[0.82,1.64]$ \\
\hline & & 2.3.2 Cohort studies (crude RR) & 5 & $0.71[0.49,1.04]$ \\
\hline & & 2.3.3 Cohort studies (adjusted RR) & 3 & $0.56[0.46,0.67]$ \\
\hline \multirow[t]{8}{*}{3 Main wall material ${ }^{2}$} & 3.1 Anaemia in children aged $0-11$ years & 3.1.1 Case-control, cross-sectional and cohort studies (crude OR) & 1 & $0.58[0.33,1.02]$ \\
\hline & & 3.1.2 Case-control, cross-sectional and cohort studies (adjusted OR) & 1 & $0.57[0.29,1.12]$ \\
\hline & 3.2 Malaria infection & 3.2.1 Case-control, cross-sectional and cohort studies (crude OR) & 12 & $0.57[0.42,0.78]$ \\
\hline & & 3.2.2 Case-control, cross-sectional and cohort studies (adjusted OR) & 7 & $0.73[0.62,0.85]$ \\
\hline & 3.3 Clinical malaria & 3.3.1 Case-control and cross-sectional studies (crude OR) & 7 & $0.63[0.43,0.93]$ \\
\hline & & 3.3.2 Case-control and cross-sectional studies (adjusted OR) & 1 & $0.16[0.06,0.44]$ \\
\hline & & 3.3.3 Cohort studies (crude RR) & 1 & $2.07[1.18,3.63]$ \\
\hline & & 3.3.4 Cohort studies (adjusted RR) & 2 & $1.05[0.48,2.30]$ \\
\hline \multirow[t]{7}{*}{4 Main roof material ${ }^{2}$} & 4.1 Anaemia in children aged $0-11$ years & 4.1.1 Case-control, cross-sectional and cohort studies (crude OR) & 1 & $0.71[0.45,1.12]$ \\
\hline & 4.2 Malaria infection & 4.2.1 Case-control, cross-sectional and cohort studies (crude OR) & 9 & $0.64[0.48,0.86]$ \\
\hline & & 4.2.2 Case-control, cross-sectional and cohort studies (adjusted OR) & 6 & $0.83[0.64,1.08]$ \\
\hline & 4.3 Clinical malaria & 4.3.1 Case-control and cross-sectional studies (crude OR) & 4 & $0.86[0.48,1.53]$ \\
\hline & & 4.3.2 Case-control and cross-sectional studies (adjusted OR) & 1 & $0.30[0.13,0.66]$ \\
\hline & & 4.3.3 Cohort studies (crude RR) & 2 & $0.59[0.52,0.67]$ \\
\hline & & 4.3.4 Cohort studies (adjusted RR) & 3 & $0.79[0.70,0.88]$ \\
\hline \multirow[t]{2}{*}{5 Main floor material ${ }^{2}$} & 5.1 Anaemia in children aged $0-11$ years & 5.1.1 Case-control, cross-sectional and cohort studies (crude OR) & 1 & $0.78[0.45,1.34]$ \\
\hline & 5.2 Malaria infection & 5.2.1 Case-control, cross-sectional and cohort studies (crude OR) & 1 & $1.20[0.69,2.09]$ \\
\hline
\end{tabular}


Table 2 Summary of findings of meta-analyses of the association between specific house features and malaria (Continued)

\begin{tabular}{|c|c|c|c|c|}
\hline & & 5.2.2 Case-control, cross-sectional and cohort studies (adjusted OR) & 2 & $0.74[0.57,0.96]$ \\
\hline & 5.3 Clinical malaria & 5.3.1 Case-control and cross-sectional studies (crude OR) & 1 & $0.19[0.06,0.57]$ \\
\hline & & 5.3.2 Cohort studies (adjusted RR) & 1 & $0.81[0.62,1.06]$ \\
\hline \multirow[t]{6}{*}{6 Eaves $^{3}$} & 6.1 Malaria infection & 6.1.1 Case-control, cross-sectional and cohort studies (crude OR) & 4 & $0.70[0.58,0.84]$ \\
\hline & & 6.1.2 Case-control, cross-sectional and cohort studies (adjusted OR) & 3 & $0.78[0.70,0.87]$ \\
\hline & 6.2 Clinical malaria & 6.2.1 Case-control and cross-sectional studies (crude OR) & 5 & $0.76[0.55,1.07]$ \\
\hline & & 6.2.2 Case-control and cross-sectional studies (adjusted OR) & 1 & $0.53[0.36,0.80]$ \\
\hline & & 6.2.3 Cohort studies (crude RR) & 1 & $0.75[0.50,1.12]$ \\
\hline & & 6.2.4 Cohort studies (adjusted RR) & 2 & $0.71[0.46,1.11]$ \\
\hline \multirow[t]{2}{*}{7 Ceiling $^{4}$} & 7.1 Clinical malaria & 7.1.1 Case-control and cross-sectional studies (crude OR) & 3 & $0.68[0.56,0.83]$ \\
\hline & & 7.1.2 Case-control and cross-sectional studies (adjusted OR) & 1 & $0.65[0.46,0.93]$ \\
\hline 8 Elevation ${ }^{5}$ & 8.1 Malaria infection & 8.1.1 Case-control and cross-sectional studies (crude OR) & 1 & $1.00[1.00,1.00]$ \\
\hline
\end{tabular}

${ }^{1}$ Screened versus unscreened; ${ }^{2}$ Modern versus traditional main wall, roof and floor material: traditional homes were considered to have mud walls, a thatched roof and earth floors in sub-Saharan Africa (except in areas of high rainfall including Equatorial Guinea, where the basic wall material is typically concrete or wood [18]); mud or stone walls, a thatched, wood or mud roof and earth floors in North Africa; wood or bamboo walls, a thatched roof and wooden (stilted) floors in Southeast Asia; mud or wood walls, a thatched roof and earth or wooden (stilted) floors in South Asia; adobe or mud and wood walls, a thatched roof and earth floors in South America. ${ }^{3}$ Closed versus open eaves; ${ }^{4}$ Presence versus absence of a ceiling; ${ }^{5}$ Elevated versus non-elevated houses 


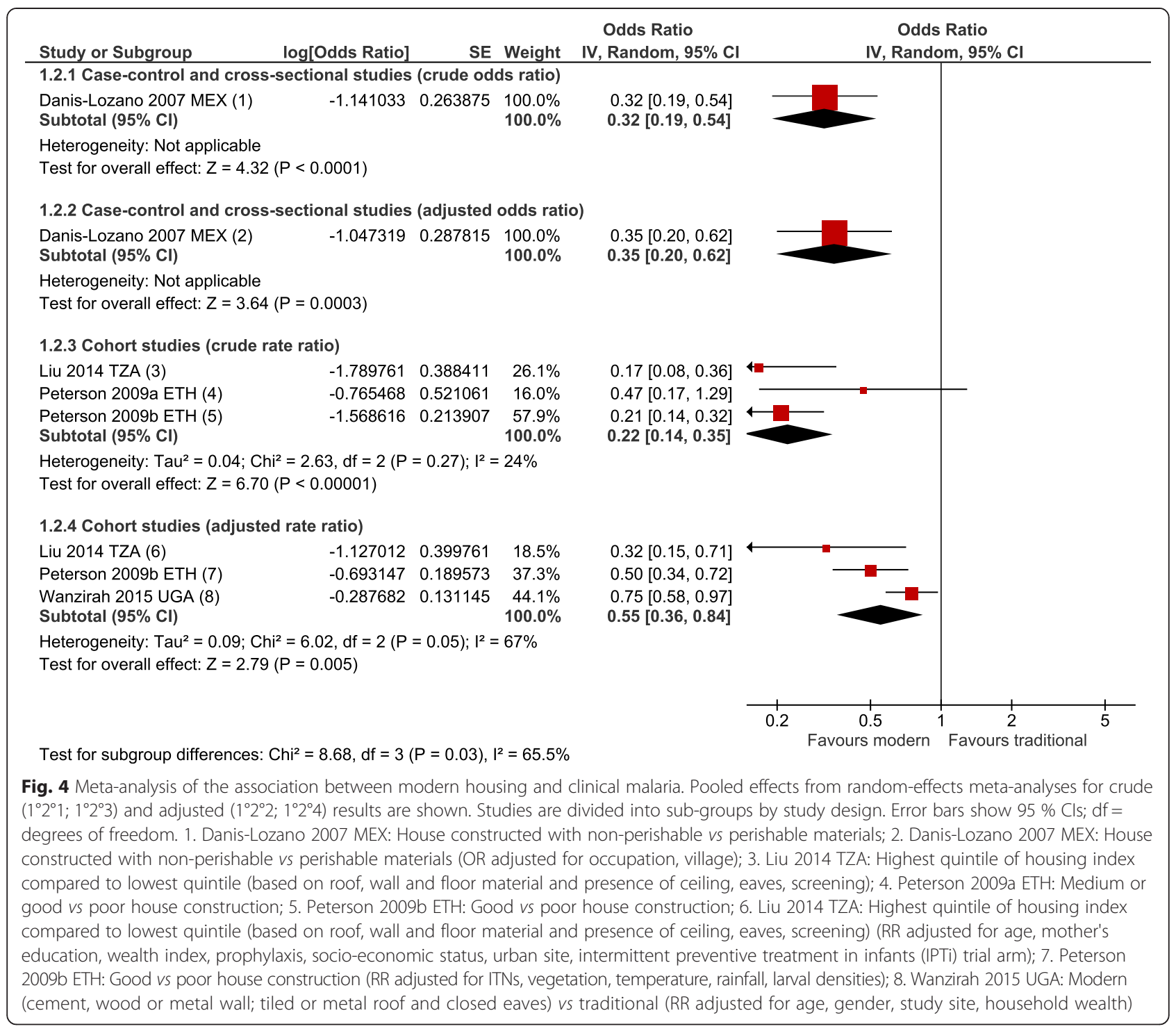

The presence of a ceiling possibly replicates the protective effect of closed eaves. Conversely, eaves may be less important in South East Asia, where vector entry differs. For example, open verandas are a key feature for house entry by Anopheles philippinensis in Laos PDR [20]. Screening doors and windows can help to directly block vector entry, while modern wall and roof materials may contain fewer gaps, alter the attractiveness of the interior environment to mosquitoes or provide fewer resting sites for mosquitoes than traditional materials such as mud or thatch. It has also been hypothesized that metalroofed homes are hotter and less conductive for mosquito survival; in Tanzania, the mean physiological age of vectors and sporozoite rate was observed to be lower in more modern versus traditional villages [31]. Understanding the mechanism of protection of different house features against individual vectors is important for identifying potential synergy or discordance with IRS and LLINs.

Housing is incrementally improving across much of SSA as living standards increase. The present analysis suggests that modern house improvements should be further evaluated in relation to malaria, in addition to specific house modifications including screening. If effective, housing could help reduce reliance on insecticides by providing an additional and more permanent intervention where LLINs and IRS are compromised by behavioural and physiological resistant vectors [2]. Furthermore, since malaria has declined in many African countries, often prior to specific intervention, further research to evaluate the contribution of housing improvements and the expansion of urban environments less conducive to malaria transmission is advocated [5]. Improving the home environment aligns with integrated 
vector management (IVM) and may help protect against other vector-borne diseases, such as filariasis, cutaneous leishmaniasis, Japanese encephalitis, and dengue, where vectors enter houses [32], and diarrhoeal disease, through better water, sanitation and hygiene (WASH). Since global housing programmes are key strategies of UN-HABITAT and Habitat for Humanity among other organisations, a pipeline for building malaria-safe homes already exists.

Improving housing will not be equally effective everywhere, since outdoor transmission can limit the efficacy of interventions centered on the home. Future research should address questions of equity by investigating whether mosquitoes diverted from improved houses may increase exposure among unprotected neighbours. Potentially damaging health effects must also be considered, such as an increased risk of respiratory diseases if airflow is restricted in the presence of certain cooking fuels. It is also shown here that the evidence base for housing needs strengthening, with only one intervention study that measured clinical outcomes [11]. Therefore further small-scale experimental studies to pinpoint exactly which house features can reduce vector entry cost effectively in different settings, RCTs with epidemiological outcomes, and concurrent studies addressing how to incorporate protective features into local house designs and building regulations are needed.

\section{Conclusions}

Despite low quality evidence, the direction and consistency of effects indicate that housing is an important risk factor for malaria. Future research should evaluate the protective effect of both specific house features and incremental housing improvements associated with socio-economic development. Investment in such research and in housing programmes should be considered a natural component of malaria control efforts and a close complement to IVM and WASH as part of long-term, sustainable development.

\section{Additional files}

\section{Additional file 1: Search strategy. \\ Additional file 2: Characteristics of included studies. \\ Additional file 3: Risk of bias assessment for studies included in the quantitative analysis. \\ Additional file 4: Funnel plots to assess publication bias. \\ Additional file 5: Association between house construction and entomological outcomes.}

\section{Abbreviations}

$\mathrm{Cl}$ : Confidence interval; EIR: Entomological inoculation rate; EPOC: Cochrane effective practice and organization of care; GRADE: Grading of recommendations, assessment, development and evaluation; HBR: Human biting rate; IRS: Indoor residual spraying; IVM: Integrated vector management; LLIN: Long-lasting insecticidal net; OR: Odds ratio; RCT: Randomized controlled trial; RDT: Rapid diagnostic test; RR: Rate ratio; SSA: Sub-Saharan Africa; WASH: Water, sanitation and hygiene.

\section{Competing interests}

The authors declare that they have no competing interests. The study sponsors had no role in study design, in the collection, analysis and interpretation of data, in writing the report, and in the decision to submit for publication.

\section{Authors' contributions}

$S W L, R G, G D$, and IK conceived of the study. All authors developed the study design. LST and MI searched the literature. LST extracted and analyzed the data and prepared the manuscript. IK analyzed the entomology data. BW advised on the systematic review and meta-analysis. All authors had access to study data and reviewed the final manuscript. LST and SWL had final responsibility for the decision to submit for publication. All authors read and approved the final manuscript.

\section{Author's information}

Lucy S Tusting and Matthew M Ippolito are joint first authors.

\section{Acknowledgements}

This work was supported by the Malaria Centre at the London School of Hygiene \& Tropical Medicine; Leverhulme Centre for Integrative Research in Agriculture and Health; Research and Policy for Infectious Disease Dynamics (RAPIDD) program of the Science and Technology Directorate, US Department of Homeland Security, the Fogarty International Center (US National Institutes of Health); the Bill \& Melinda Gates Foundation (OPP1053338); Novartis Foundation for Sustainable Development; Medical Research Council and Department For International Development. We thank Yanfang Jiang and Margaux Mesle for their help translating papers.

\section{Author details}

${ }^{1}$ Department of Disease Control, London School of Hygiene \& Tropical Medicine, London WC1E 7HT, UK. ²Department of Medicine, Division of Infectious Diseases, Johns Hopkins University School of Medicine, 1830 Building Room 450B, 600 North Wolfe Street, Baltimore, MD 21287, USA. ${ }^{3}$ Department of Infectious Disease Epidemiology, London School of Hygiene \& Tropical Medicine, London, UK. ${ }^{4}$ Department of Medicine, University of California, San Francisco, CA 94143, USA. ${ }^{5}$ Global Health Group, University of California, San Francisco, CA 94105, USA. ${ }^{6}$ School of Biological and

Biomedical Sciences, Durham University, Durham DH1 3LE, UK.

Received: 12 January 2015 Accepted: 6 May 2015

Published online: 09 June 2015

\section{References}

1. WHO. World Malaria Report. Geneva: World Health Organization; 2014.

2. Edi CVA, Koudou BG, Jones CM, Weetman D, Ranson H. Multiple-insecticide resistance in Anopheles gambiae mosquitoes, southern Côte d'Ivoire. Emerg Infect Dis. 2012;18:1508-11.

3. Fegan G, Noor A, Akhwale W, Cousens S, Snow R. Effect of expanded insecticide-treated bednet coverage on child survival in rural Kenya: a longitudinal study. Lancet. 2007;370:1035-9.

4. Ceesay S, Casals-Pascual C, Nwakanma D, Walther M, Gomez-Escobar N, Fulford A, et al. Continued decline of malaria in The Gambia with implications for elimination. PLoS One. 2010;5:e12242.

5. Tusting LS, Willey B, Lucas H, Thompson J, Kafy HT, Smith R, et al. Socioeconomic development as an intervention against malaria: a systematic review and meta-analysis. Lancet. 2013;382:834.

6. Anderson L, Simpson D, Stephens M. Durable housing improvements to fight malaria transmission: Can we learn new strategies from past experience? Atlanta: Habitat for Humanity International Global Programs Department; 2014

7. Huho B, Briët O, Seyoum A, Sikaala C, Bayoh N, Gimnig J, et al. Consistently high estimates for the proportion of human exposure to malaria vector populations occurring indoors in rural Africa. Int J Epidemiol. 2013;42:235-47.

8. Celli A: The new prophylaxis against malaria. J Trop Med. 1901, 119-23

9. Hackett LW, Missirolli A. Housing as a factor in malaria control. Trans R Soc Trop Med Hyg. 1932;26:65-72.

10. Wanzirah H, Tusting LS, Arinaitwe E, Katureebe A, Maxwell K, Rek J, et al. Mind the gap: house construction and the risk of malaria in Ugandan children. PLoS One. 2015;10:e0117396

11. Kirby M, Ameh D, Bottomley C, Green C, Jawara M, Milligan P, et al. Effect of two different house screening interventions on exposure to malaria vectors 
and on anaemia in children in The Gambia: a randomised controlled trial. Lancet. 2009;374:998-1009.

12. UNCTAD. Economic development in Africa report 2014: catalysing investment for transformative growth in Africa. Geneva: United Nations Conference on Trade and Development; 2014.

13. UN-Habitat: State of African Cities 2010. Governance, inequalities and urban land markets. Nairobi: UN Habitat; 2010.

14. RBM/UNDP. Multisectoral action framework for malaria. Geneva: Roll Back Malaria/United Nations Development Programme; 2013.

15. Stroup DF, Berlin JA, Morton SC, Olkin I, Williamson GD, Rennie D, et al. Meta-analysis of observational studies in epidemiology. JAMA. 2000;283:2008-12.

16. Modher D, Liberati A, Tetzlaff J, Altman DG, Group TP. Preferred reporting items for systematic reviews and meta-analyses: The PRISMA Statement. PLoS Med. 2009;6:e1000097.

17. Ippolito M, Tusting LS, Gosling R, Dorsey G, Lindsay SW. Malaria and housing: a systematic review and meta-analysis. PROSPERO, 2014: CRD42014009048.

18. Bradley J, Rehman AM, Schwabe C, Vargas D, Monti F, Ela C, et al. Reduced prevalence of malaria infection in children living in houses with window screening or closed eaves on Bioko Island, Equatorial Guinea. PLoS ONE. 2013;8:e80626.

19. UN-Habitat. Tunisia urban housing sector profile. Nairobi: UN-Habitat; 2011.

20. Hiscox A, Khammanithong P, Kaul S, Sananikhom P, Luthi R, Hill N, et al. Risk factors for mosquito house entry in the Lao PDR. PLoS One. 2013;8:e62769.

21. Guthmann JP, Hall AJ, Jaffar S, Palacios A, Lines J, Llanos-Cuentas A. Environmental risk factors for clinical malaria: a case-control study in the Grau region of Peru. Trans R Soc Trop Med Hyg. 2001;95:577-83.

22. Cochrane Collaboration. Suggested risk of bias criteria for EPOC reviews. Oxford: Cochrane Collaboration; 2013.

23. Wells G, Shea B, O'Connell D, Peterson J, Welch V, Losos M, et al. The Newcastle-Ottawa Scale (NOS) for assessing the quality of nonrandomised studies in meta-analyses. Ottawa: University of Ottawa.

24. Harbord R, Egger M, Sterne J. A modified test for small-study effects in meta-analyses of controlled trials with binary endpoints. Stat Med. 2006;25:3443-57.

25. GRADE Working Group. Grading quality of evidence and strength of recommendations. BMJ. 2004;328:1490-4.

26. Massebo F, Lindtjorn B. The effect of screening doors and windows on indoor density of Anopheles arabiensis in south-west Ethiopia: a randomized trial. Malar J. 2013;12:1.

27. Kampango A, Braganca M, Sousa BD, Charlwood JD. Netting barriers to prevent mosquito entry into houses in southern Mozambique: a pilot study. Malar J. 2013;12:1.

28. Mng'ong'o FC, Sambali JJ, Sabas E, Rubanga J, Magoma J, Ntamatungiro AJ, et al. Repellent plants provide affordable natural screening to prevent mosquito house entry in tropical rural settings-results from a pilot efficacy study. PLoS One. 2011;6:10.

29. Kirby MJ, Njie M, Dilger E, Lindsay SW. Importance of eaves to house entry by anopheline, but not culicine, mosquitoes. J Med Entomol. 2009;46:505-10

30. Ogoma SB, Lweitoijera DW, Ngonyani H, Furer B, Russell TL, Mukabana WR, et al. Screening mosquito house entry points as a potential method for integrated control of endophagic filariasis, arbovirus and malaria vectors. PLoS Negl Trop Dis. 2010;4:8.

31. Magesa SM, Wilkes TJ, Mnzava AE, Njunwa KJ, Myamba J, Kivuyo MD, et al. Trial of pyrethroid impregnated bednets in an area of Tanzania holoendemic for malaria. Part 2. Effects on the malaria vector population. Acta Trop. 1991:49:97-108.

32. Wilson AL, Dhiman R, Kitron U, Scott TW, van den Berg H, Lindsay SW. Benefit of insecticide-treated nets, curtains and screening on vector borne diseases, excluding malaria: a systematic review and meta-analysis. PLoS Negl Trop Dis. 2014;8:e3228.

33. ICF International. Demographic and Health surveys (various). Calverton, ICF International, 2014

34. Liu JX, Bousema T, Zelman B, Gesase S, Hashim R, Maxwell C, et al. Is housing quality associated with malaria incidence among young children and mosquito vector numbers? Evidence from Korogwe, Tanzania. PLoS ONE. 2014;9:e87358.

35. ICF International. The DHS progam STATcompiler. Calverton: ICF International; 2015

\section{Submit your next manuscript to BioMed Central and take full advantage of:}

- Convenient online submission

- Thorough peer review

- No space constraints or color figure charges

- Immediate publication on acceptance

- Inclusion in PubMed, CAS, Scopus and Google Scholar

- Research which is freely available for redistribution

Submit your manuscript at www.biomedcentral.com/submit 